\title{
Pain and delirium: mechanisms, assessment, and management
}

\author{
Elizabeth L. Sampson ${ }^{1,2}$ (D) Emily West ${ }^{1} \cdot$ Thomas Fischer $^{3}$ (])
}

Received: 18 October 2019 / Accepted: 10 December 2019 / Published online: 9 January 2020

(c) The Author(s) 2020

\section{Key summary points}

Aim To provide a clinical overview of the association between pain and delirium, the mechanisms which may underlie this and how pain can be detected and managed in delirium.

Findings There are few studies in this field. It is likely that pain is a key cause of delirium but this relationship is complex and mediated by many factors. Observational tools may help diagnose pain in delirium but need further testing.

Message Pain is a potentially treatable cause of delirium and future research in how to better detect and managed this is required.

\begin{abstract}
Purpose Pain and delirium are common problems for older people. Both conditions are prevalent in acute hospital settings. In people living in the community, delirium often precipitates presentation to the emergency department. Pain and delirium are known to interact in a complex and multidirectional way. This can make it challenging for staff to recognize and treat pain in people with delirium.

Methods This paper aims to explore the complex relationship between pain and delirium and on pain assessment in delirium, drawing together evidence from a range of settings including acute medical, cardiac and orthopaedic post-operative cohorts, as well as from aged care.

Results A limited number of studies suggest there is an association between pain and delirium; however, this is a complex, particularly where analgesics which may-themselves cause delirium are prescribed. Factors acting on the pathway between pain and delirium may include depression, sleep deprivation and disturbance of the cholinergic system. Delirium affects the ability to self-report pain. The fluctuating nature of delirium as well as reduced awareness and attention may challenge practitioners in recognizing, assessing and treating pain. Evidence concerning the reliability and validity of current observational and self-assessment tools in people with delirium is unclear but some show promise in this population.

Conclusion The current evidence base regarding assessing pain in people with delirium is lacking. Tentative recommendations, drawing on current guidelines require robust testing. Guidelines for people with pain and dementia require adaptations regarding the unique characteristics of delirium. The complex interplay between dementia, pain and delirium warrants further investigation across a range of settings.
\end{abstract}

Keywords Delirium $\cdot$ Pain $\cdot$ Pain measurement $\cdot$ Analgesics $\cdot$ Hospitals $\cdot$ Care homes

\section{Introduction}

Elizabeth L. Sampson

e.sampson@ucl.ac.uk

1 Marie Curie Palliative Care Research Department, Division of Psychiatry, University College London, London, UK

2 Barnet Enfield, Haringey Mental Health Trust Liaison Psychiatry Team, North Middlesex University Hospital, London, UK

3 Evangelische Hochschule Dresden (ehs), Dresden, Germany
Both pain and delirium are common problems for older people across a range of clinical and residential settings. Pain affects $20-46 \%$ of older people living in the community, and $28-73 \%$ of those living in residential care. Chronic pain rates are reported to be even higher [1]. The prevalence of delirium in the community is low, but a delirium prevalence of $36.8 \%$ has been reported in residential aged care settings [2] and the onset of delirium often precipitates presentation to 
the emergency department. Here, $8-17 \%$ of all older people and $40 \%$ of those residing in residential care have delirium. In general, medical and geriatric hospital units' delirium is found in 29-64\% of inpatients [3]. Pain and delirium often occur together [4] especially in older populations and those with dementia.

Interactions between pain and delirium are complex and multidirectional. While pain may be a risk factor for delirium in older persons, analgesics given for pain management may also increase the risk of delirium [4, 5]. However, the complex interplay of pain, pain management and delirium in older persons remains unclear. In this article, we present a broad clinical overview of the topic, with particular reference to the care of older people, using recent evidence from other populations to understand some of these complexities. We will review the current evidence on pain assessment strategies in adults, and present a summary of guidance on pain management in the context of delirium to inform clinical practice and research.

\section{The nature of pain}

Pain is defined as "an unpleasant sensory and emotional experience associated with actual or potential tissue damage or described in terms of such damage". Because it is highly subjective, pain is best assessed via self-report - this is considered the "gold standard" of pain assessment [6]. Delirium can interfere with a person's ability to self-report pain, due to disturbances in attention and awareness that develop over a short period of time and tend to fluctuate. Additional disturbances affect memory, orientation, language, perception and other areas [7].

Pain is a complex phenomenon consisting not only of the sensory component, which refers to where a person locates pain in their body and how they describe painful sensations and pain intensity. There is also a significant emotional component, which refers to feelings and affects associated with painful experiences. A further cognitive component reflects how a person attributes meaning to the painful experience, taking into account expectations and past experiences [8]. The experience of pain is also influenced by past and current social interactions between the person in pain and their carers or clinicians. In addition to this, discomfort must be considered. This is different to "pure" nociceptive pain and has a wide range of causes such as poor positioning, feeling too hot or cold, hunger, thirst or needing to go to the toilet.

\section{The association between pain and delirium}

Given the risk factors for both conditions, pain and delirium will commonly co-exist. However, there is limited data exploring this across settings. The current evidence base mostly centers on elective orthopaedic or cardiac post-operative surgical cohorts. Table 1 gives an overview of studies that have examined this association.

The literature consistently suggests an association between pain and delirium, even after controlling for potential confounders of this relationship such as age [9] comorbidity [10] and severity of dementia. Some authors have noted a "dose-response" gradient in this association, with increasing pain severity increasing the risk of both sub-syndromal [11] and full-syndromal delirium [12], although this is not a consistent finding.

When considering causality it is important to examine the temporal precedence of findings. Prospective methods, which measure pain and delirium, both pre- and post-operatively [10] will provide more robust evidence than crosssectional studies. Whether analgesics increase delirium risk through their side effects, or reduce delirium risk by decreasing pain is a particularly interesting question as both under-treatment and over-treatment of pain can precipitate delirium. Most studies have focused on ICU populations who are often prescribed sedatives in conjunction with analgesia. The evidence in older "geriatric" populations is inconclusive, and mostly from small-scale studies of surgical cohorts. In post-operative older populations, Vaurio et al. [9] found that oral narcotic analgesics reduced delirium risk when compared to IV and epidural analgesics. They suggest that this may be due to oral opioid analgesics resulting in lower blood drug levels. It is possible that patients receiving IV analgesics may have initially been in more pain; however, the study did control for initial pain severity in analysis.

A systematic review of the association between opioids and delirium in older populations found moderate quality evidence suggesting that where acute severe pain occurs (e.g., hip fracture) lower doses of opioids may paradoxically be associated with a higher risk of delirium. This supports the concept of acute, severe pain as an important contributing factor to delirium [5] and suggests that treating pain with adequate doses of opioids may lower delirium risk.

\section{Understanding cause and effect}

Delirium is caused by multiple stressors including infection, inflammation, drug toxicity and metabolic abnormalities-all of which lead to an acute cerebral stress response. Pain has a similar effect, acutely inducing the release of catecholamines and a short-term pro-inflammatory sympathetic response [16]. Chronic pain leads to dysfunction of the cortisol system, and prolonged over-activity of inflammatory cytokines. Therefore, acute or chronic pain may lower the threshold for the development of delirium. This is particularly notable in older people with concomitant frailty, acute physical illness or dementia, who have a reduced cognitive reserve. It is possible that discomfort, whether acute 


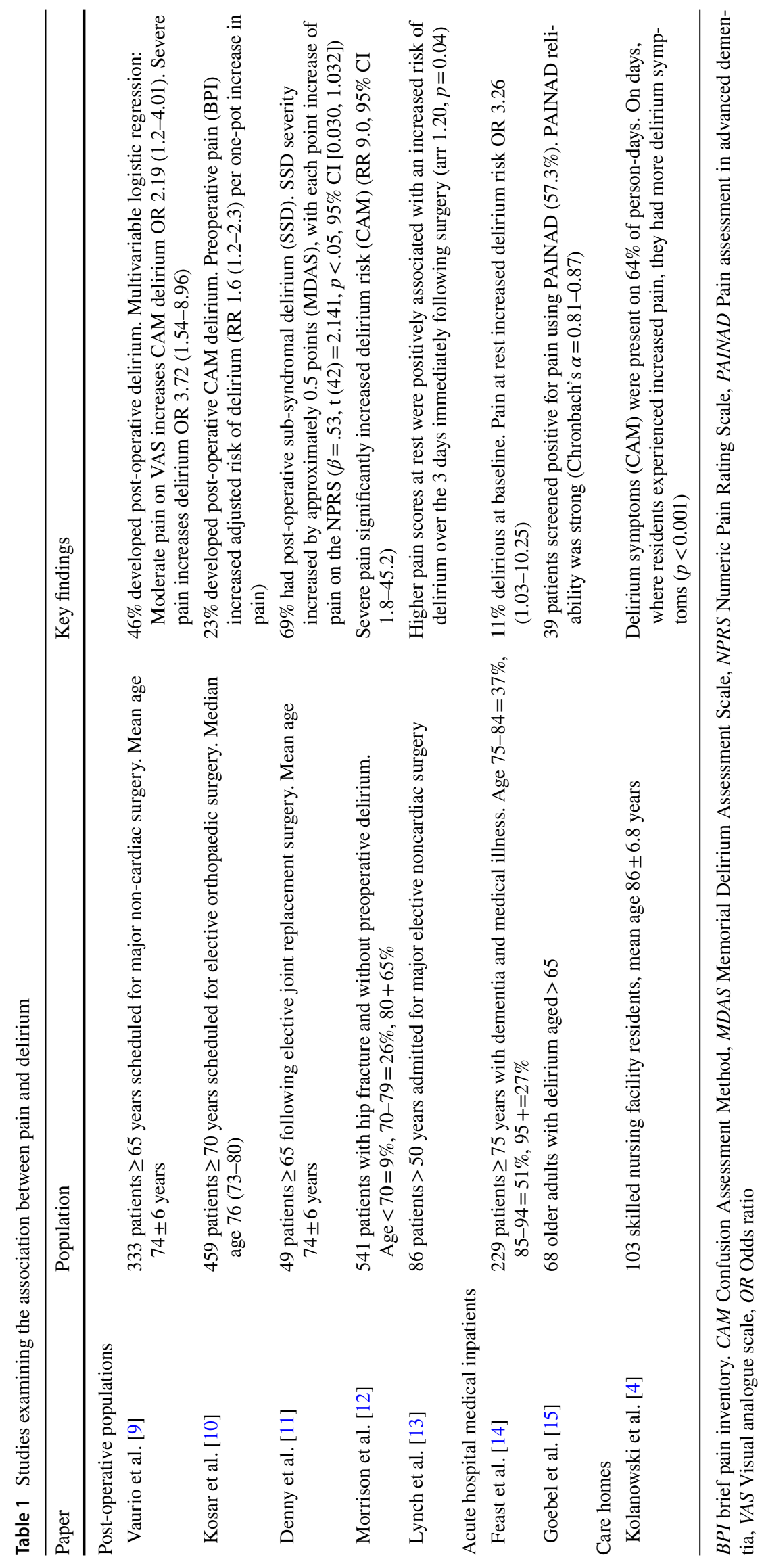




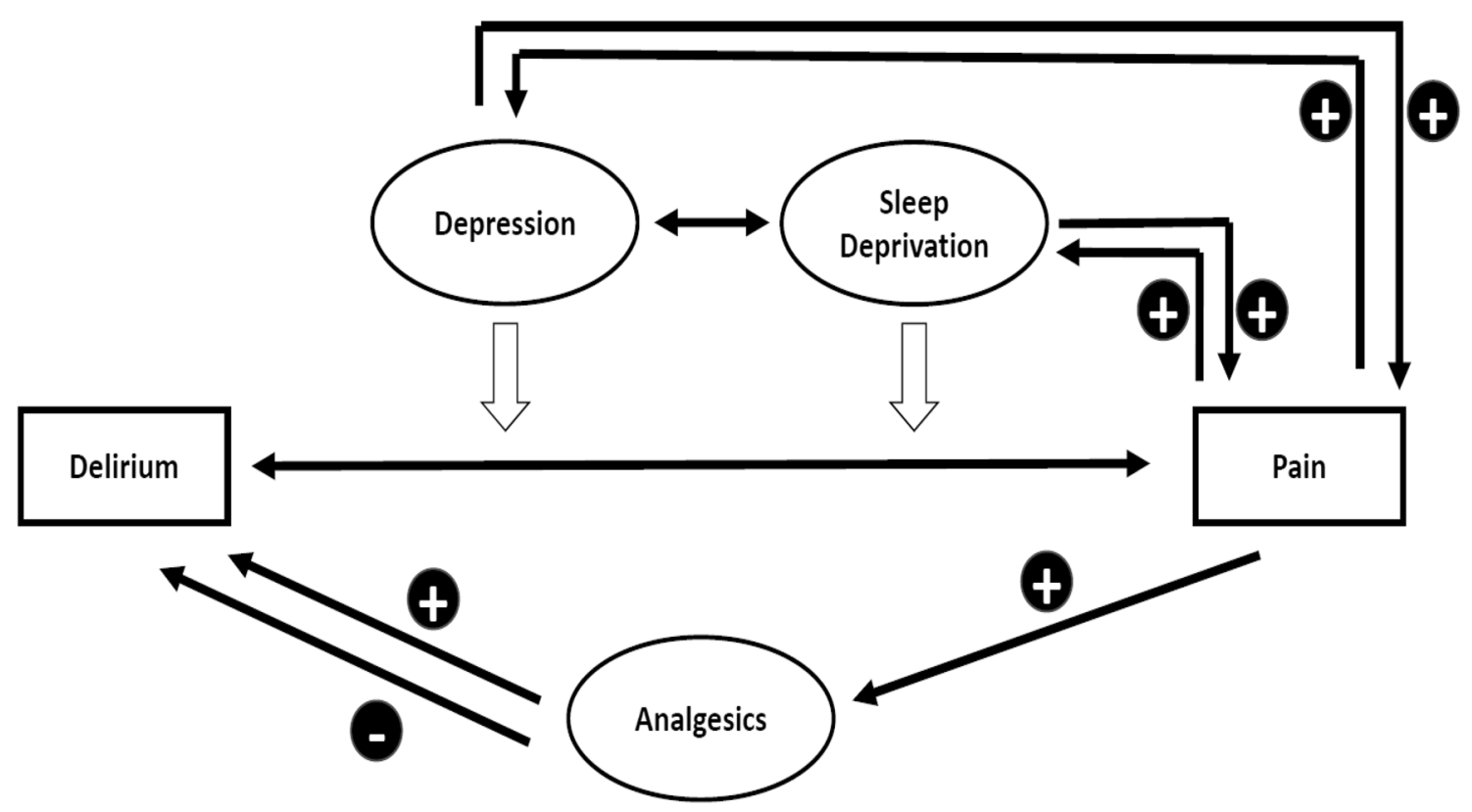

Fig. 1 Association between delirium, pain and selected contributing factors

or chronic may also cause a similar stress reaction that may precipitate delirium, although no studies to date have examined this.

The causal association between pain and delirium is highly complex, and may be bi-directional. The cholinergic system may be a key link. A reduction in central cholinergic activity is the final common pathway in the development of symptoms of delirium, but the cholinergic system also has a vital role in the modulation of pain. In animal models, decreasing acetylcholine levels or activity potentiates nociceptive sensitivity and can induce hyperalgesia or allodynia. Acetylcholinesterase inhibitors such as Donepezil have a dose-dependent analgesic effect in humans. However, there is also developing evidence that chronic pain states modulate cholinergic activity [17]; further study of this dynamic interaction may help us with future understanding of how pain may precipitate delirium.

In addition, a multitude of clinical factors may mediate the association between pain and delirium. Here we consider two exemplar conditions, depression and sleep deprivation. Depression is common in older people and also a risk factor for delirium. Pain is also known to substantially increase the occurrence and severity of depression [18]. In a robustly conducted prospective cohort study, Kosar et al. [10] demonstrated that preoperative pain and depressive symptoms were independently associated with the development of postoperative delirium. In addition to this, there was significant interaction, with pain increasing the risk of delirium further in those with depression.

There is also strong evidence (mainly from ICU populations) that sleep deprivation is a risk factor for the development and subsequent severity of delirium-where disruption of circadian rhythms is a key symptom. Lower melatonin levels are also associated with an increased delirium risk. It may seem obvious that pain disrupts sleep, but there is also an increasing body of evidence suggesting that sleep deprivation induces a hyper-algesic response [19]. Inflammation and, particularly, pro-inflammatory cytokines are key potential mediators of this association.

All of the factors we have discussed are interrelated, and subject to multiple and complex interactions with each other. For example, chronic depression will disrupt the sleep-wake cycle_-both of these factors are exacerbated by pain, and both are well-established risk factors for delirium. This highlights the necessity of detailed, comprehensive and holistic assessment of the person with pain and delirium, and the construction of tailored multi-component management strategies (Fig. 1).

\section{The impact of delirium on the expression of pain}

Delirium may impact how pain is expressed, which in turn affects the pain assessment process. This can manifest in several ways including through altered levels of attention and awareness, as well as the fluctuating and sometimes prolonged nature of delirium.

\section{Altered levels of attention and awareness}

Delirium occurs along a spectrum, from hypoactive to hyperactive. Both presentations can make pain assessment challenging. Clinicians may tend to interpret signs of pain 
more readily in hyperactive delirium as opposed to hypoactive delirium, where people are usually withdrawn and silent. Gagnon et al. [20] argue that hyperactive delirium may lead to more breakthrough pain, when compared to hypoactive delirium, due to frequent, intense or purposeless movement. Hyperactive delirium may, therefore, exacerbate movement-related pain.

There is some indication that reduced levels of attention and awareness in hypoactive delirium mean that people with delirium are less likely to initiate communication [21] and altered awareness may directly impair how pain is perceived. Therefore, clinicians need to be proactive in starting conversations about pain in people with delirium.

\section{Fluctuation and duration of delirium}

Sudden onset of delirium may rapidly change the person's ability to participate in the pain assessment process. Pain assessment strategies need to be adapted to respond to newly altered levels of awareness and attention and to take into account new and changeable symptoms. As these fluctuate, so can the person's ability to participate in the pain assessment process. Fluctuations may occur rapidly, necessitating the use of a range of different pain assessment strategies in a short period of time. This is especially pertinent in situations, where acute pain is likely to occur, for example after surgery. Sirsch et al. [22] recommend that the person's ability to contribute to pain assessment should be evaluated prior to the commencement of a pain assessment. Herr et al. [23] recommend "serial assessments for the ability to self-report" pain for patients in intensive care settings with fluctuating delirium. Hadjistavropoulos et al. [24] suggest repeating pain assessments when delirium symptoms subside. Sudden onset and fluctuating course of delirium mean that this may need to be repeated several times a day in all patients with potential delirium. In cases of prolonged delirium, this regime may be required over an extended period of time.

\section{Behavioural signs of pain in people with delirium}

If self-reporting pain is impossible for a person with delirium, non-verbal behavioural signs of pain may become the most important aspect of pain communication-similar to what has been suggested in people with dementia. However, it is not completely clear what behaviours should be looked for. Reference is often made to The American Geriatrics Society's Panel on Persistent Pain in Older Adults overview of "common pain behaviours in older adults" [25]. This lists facial expressions, verbalisations/vocalisations, body movements, changes in interpersonal interactions, changes in activity patterns or routines, and mental status change.

The validity of this consensus-based approach has not been evaluated for people with delirium. A specific problem for pain assessment in people with delirium is the overlap of symptoms of delirium with commonly cited pain behaviours [26, 27]—especially with regard to hyperactive behaviours such as restlessness and screaming. "Agitation" caused by delirium may be confused with pain [20] by clinicians. Behavioural pain assessment instruments for people with dementia often consider agitation to be a sign of pain, though some research suggests that agitation, when considered as a symptom of hyperactive delirium, may inflate behavioural pain scores [28, 29]. Future research will need to examine whether behavioural pain signs can be identified that are less prone to confounding from hyperactive behaviours associated with delirium, potentially in the domain of facial expressions.

Two studies have asked clinicians what they perceived to be behavioural pain signs in patients with delirium-one regarding post-operative older patients [30], and one palliative care patients [31]. These identified vocalisations and changes in facial expression as being key expressions of pain (see Table 2).

Table 2 Possible behavioural pain signs in people with delirium

\begin{tabular}{ll}
\hline Context & Indicator \\
\hline $\begin{array}{l}\text { Post-operative older persons } \\
\text { [33] }\end{array}$ & Change in usual behaviour (reluctant to move; patient is not acting right (family input); quiet; irritable; agita- \\
& tion (see also: non-verbal cues); reluctant to do anything \\
& Non-verbal cues/behaviour (guarding the affected side; restless; agitation (see also: Change in usual behaviour) \\
& Facial expression (grimacing, frowning, clenching jaws, gloomy face) \\
& Vocalisations (sighing, moaning, crying, yelling) \\
& Not categorised (elevated vital signs; reluctant to be touched; reluctant to deep breath and cough; points to \\
& where hurts; quivering; whiny) \\
& Moaning \\
Groaning & Grimacing
\end{tabular}




\section{Standardised tools for pain assessment in people with delirium}

A recent scoping review [32] identified different strategies for pain assessment in persons with delirium; including self-report using standardised tools (e.g., Numerical Rating Scales, Verbal Rating Scales), proxy reports by relatives and carers, behavioural observations, analgesic trials and combinations thereof. However, these approaches have never been systematically studied in people with delirium, and therefore, evidence for their reliability and validity is lacking. This also applies to standardised observational pain instruments developed for persons with dementia (e.g., PAINAD, Abbey Painscale or Doloplus). For patients with delirium in intensive care settings, preliminary testing showed promising results for the Critical Care Pain Observation Tool (CPOT) and the Behavioural Pain Scale for non-intubated patients.

\section{Technological Interventions}

The development of electronic and computerised pain tools has accelerated in recent years. Early tools often concentrated on developing self- or medical team assessment [33], recording and promotion of information-sharing regarding pain. Recent advances in artificial intelligence and machine learning have accelerated the development of automated pain assessment tools, which have shown promising preliminary results in elderly populations.

An example of a system currently being used in residential care settings is PainChek. This uses a combination of automated facial recognition and analysis (AFRA), alongside advanced computing methods that allow facial movements to be rapidly recorded and analysed [34], based on the Facial Action Coding System (FACS). Other elements of behavioural domains of pain are then analysed to create a comprehensive assessment. This tool has shown promise in initial trials, both in identifying pain and facilitating the identification of pain temporality. FACS has been tested in populations with dementia, including non-verbal patients [35], though more specific testing in populations with severe dementia may be warranted. This tool and AFRA have so far, not been tested in people with delirium but this may be a promising approach for future care.

\section{Recommendations for the assessment of pain in people with delirium}

Evidence regarding pain assessment strategies and instruments for people with delirium is lacking. Only tentative practice recommendations can be made, all drawn from existing guidelines but needing robust testing in practice [32]:
- A comprehensive assessment of the person, as is standard practice for all with severe illness, forms the foundation for the investigation of pain. This should include a collateral history and a physical examination with a specific focus on causes of potential or actual chronic or acute pain. The exact contents of this assessment will vary between settings (intensive, geriatric, palliative care etc.) and it should be adjusted to the individual person's needs and situation.

- All potential causes for pain, as identified in the comprehensive assessment, need to be taken into consideration when a judgement about the person's pain situation is made.

- When pain is likely, e.g., due to identified pathologies (e.g., trauma) or painful procedures (e.g., surgery) pain treatment should be initiated to avoid suffering and negative pain-related outcomes.

- The ability to provide self-report of pain needs to be assessed before a strategy for pain assessment is chosen. Due to the fluctuating nature of delirium, the ability to self-report should be re-assessed when pain is reassessed.

- Clinicians need to take the initiative and address pain, as people with delirium may not do so themselves even if they are in pain. People with hypoactive delirium may communicate verbal and non-verbal pain signs less than people with hyperactive delirium.

- Verbal and non-verbal signs of pain and delirium may overlap and clinicians need to be aware that this can be a source of diagnostic confusion. If behaviours are believed to be a sign of pain and analgesic treatment is subsequently initiated or increased, unchanged or even exacerbated behaviours may be an indication that the analgesic treatment compounds delirium.

- The Critical Care Pain Observation Tool (CPOT) and the Behavioural Pain Scale for non-intubated patients may be useful for people with delirium in intensive care but should be used cautiously as their validity is not established in people with other causes of cognitive impairment, heavily sedated or unconscious patients.

- Behavioural pain tools developed for people with dementia may be misleading when used in people with delirium. This is especially pertinent when they include items that describe social behaviours or that overlap pain and delirium, such as restlessness, vocal behaviours, agitated behaviours etc.

From the literature, no recommendations regarding the assessment of pain qualities beyond the mere existence of pain can be identified [32]. Pain intensity cannot be determined by any other means than self-report [36]. Pain location may be inferred from comprehensive assessment, cognition associated with the pain experience may be compromised 
by delirium, and motivational-affective aspects of the pain experience may be communicated through pain behaviours.

\section{Future directions}

Evidence around the association between pain and dementia, and how to assess and manage this complex syndrome alongside delirium is only starting to emerge. Recommendations from guidelines on pain assessment in older persons and those with dementia may indicate helpful strategies, however, the characteristics of delirium - such as altered levels of attention and awareness as well fluctuations-require adaptation of the pain assessment process and dedicated research. Strategies to enhance and support self-report about pain in people with delirium need to be explored. Furthermore, a better understanding of behavioural signs of pain and delirium, and the overlap between the two, is needed to identify specific non-verbal indicators of pain-if they exist—and improve behavioural pain tools accordingly.

People's experience of pain encompasses much more than merely sensory aspects such as intensity or localisation. Motivational-affective components may also strongly contribute to the person's experience of suffering and discomfort. However, they also offer a pathway to connect with the person and alleviate suffering. There is some indication that interventions aimed at this dimension of pain are effective in people with dementia [37], and this approach could be explored in delirium. It may have the potential to reduce prescribing of potentially deliriogenic analgesia, and aligns well with recommendations to prevent and manage delirium-for example the SIGN delirium guidance [38]. Likewise, strategies that orientate the person with delirium are recommended for management, though how this may improve pain management needs further research.

For older populations it is vital that future research takes the co-occurrence of dementia and delirium into account in studying potential interactions, and clarifying the consequences of both for pain assessment and treatment. Residential aged care and long-term care in the community, often provided by family and informal carers, must be included in research to develop strategies for pain assessment and management in people with delirium — as many older people are cared for in these settings.

To gain a better understanding of the relationship between delirium, pain and pain management, longitudinal studies in different settings are necessary. This would allow the study of causation and contributing factors. This paper provides a broad clinical overview but a more comprehensive systematic review of the association between pain and delirium in specific populations is warranted. Future research will be informed by our increasing knowledge of pathophysiological mechanisms in delirium, in particular neuroinflammation, that may promote the association between pain and delirium.
Interventional studies may reveal promising strategies to alleviate pain and delirium simultaneously, for example by carefully studying the effects of stepped-analgesic interventions - as has been demonstrated in care home residents with dementia [39]—or by complex multimodal delirium interventions, where pain assessment and management are key components [40].

Acknowledgements ELS is supported by Marie Curie Core grant funding, grant number MCCC-FCO-16-U. This paper presents work funded by the National Institute for Health Research (NIHR) under its Research for Patient Benefit (RfPB) Programme (Grant Reference Number PB-PG-0317-20019). The views expressed are those of the authors and not necessarily those of the NIHR or the Department of Health and Social Care.

Author contributions ELS and TF were invited to prepare this review and worked with EW to plan the paper, review the literature, and develop content and concepts. ELS, TF, and EW wrote the paper. All authors approved the final version.

\section{Compliance with ethical standards}

Conflict of interest The authors declare that they have no Conflict of interest.

Ethical approval This article does not contain any studies with human participants or animals performed by any of the authors. Ethical permission was not required for this review article.

Informed consent For this type of study, formal consent is not required.

Open Access This article is licensed under a Creative Commons Attribution 4.0 International License, which permits use, sharing, adaptation, distribution and reproduction in any medium or format, as long as you give appropriate credit to the original author(s) and the source, provide a link to the Creative Commons licence, and indicate if changes were made. The images or other third party material in this article are included in the article's Creative Commons licence, unless indicated otherwise in a credit line to the material. If material is not included in the article's Creative Commons licence and your intended use is not permitted by statutory regulation or exceeds the permitted use, you will need to obtain permission directly from the copyright holder. To view a copy of this licence, visit http://creativecommons.org/licenses/by/4.0/.

\section{References}

1. Abdulla A, Bone M, Adams N et al (2013) Evidence-based clinical practice guidelines on management of pain in older people. Age Ageing 42:151-153. https://doi.org/10.1093/ageing/afs199

2. Morichi V, Fedecostante M, Morandi A et al (2018) A point prevalence study of Delirium in Italian nursing homes. Dement Geriatr Cogn Disord 46:27-41. https://doi.org/10.1159/000490722

3. Inouye SK, Westendorp RG, Saczynski JS (2014) Delirium in elderly people. Lancet 383:911-922. https://doi.org/10.1016/ S0140-6736(13)60688-1

4. Kolanowski A, Mogle J, Fick DM et al (2015) Pain, delirium, and physical function in skilled nursing home patients with dementia. J Am Med Dir Assoc 16:37-40. https://doi.org/10.1016/J.JAMDA .2014.07.002 
5. Clegg A, Young JB (2011) Which medications to avoid in people at risk of delirium: a systematic review. Age Ageing 40:23-29. https://doi.org/10.1093/ageing/afq140

6. Turk D, Melzack R (2011) The measurement of pain and assessment of people experienceing pain, 2nd edn. The Guildford Press, New York

7. American Psychiatric Association. American Psychiatric Association. DSM-5 Task Force. Diagnostic and statistical manual of mental disorders : DSM-5

8. Craig KD (2015) Social communication model of pain. Pain 156:1198-1199. https://doi.org/10.1097/j.pain.000000000000018 5

9. Vaurio LE, Sands LP, Wang Y et al (2006) Postoperative delirium: the importance of pain and pain management. Anesth Analg 102:1267-1273. https://doi.org/10.1213/01.ane.0000199156 .59226.af

10. Kosar CM, Tabloski PA, Travison TG et al (2014) Effect of preoperative pain and depressive symptoms on the risk of postoperative delirium: a prospective cohort study. Lancet Psychiatry 1:431-436. https://doi.org/10.1016/S2215-0366(14)00006-6

11. Denny DL, Lindseth GN (2019) Pain, opioid intake, and delirium symptoms in adults following joint replacement surgery. West $\mathrm{J}$ Nurs Res. https://doi.org/10.1177/0193945919849096

12. Morrison RS, Magaziner J, Gilbert M et al (2003) Relationship between pain and opioid analgesics on the development of delirium following hip fracture. J Gerontol Ser A Biol Sci Med Sci 58:M76-M81. https://doi.org/10.1093/gerona/58.1.M76

13. Lynch EP, Lazor MA, Gellis JE et al (1998) The impact of postoperative pain on the development of postoperative delirium. Anesth Analg 86:781-785. https://doi.org/10.1213/00000539-19980 4000-00019

14. Feast AR, White N, Lord K et al (2018) Pain and delirium in people with dementia in the acute general hospital setting. Age Ageing 47:841-846. https://doi.org/10.1093/ageing/afy112

15. Goebel JR, Ferolito M, Gorman N (2019) Pain screening in the older adult with delirium. Pain Manag Nurs 20:519-525. https:// doi.org/10.1016/j.pmn.2019.07.003

16. Hannibal KE, Bishop MD (2014) Chronic stress, cortisol dysfunction, and pain: a psychoneuroendocrine rationale for stress management in pain rehabilitation. Phys Ther 94:1816-1825. https:// doi.org/10.2522/ptj.20130597

17. Naser PV (2018) Molecular, cellular and circuit basis of cholinergic modulation of pain. Neuroscience 387:135-148. https://doi. org/10.1016/J.NEUROSCIENCE.2017.08.049

18. Zis P, Daskalaki A, Bountouni I et al (2017) Depression and chronic pain in the elderly: links and management challenges. Clin Interv Aging 12:709-720. https://doi.org/10.2147/CIA.S113576

19. Herrero Babiloni A, De Koninck BP, Beetz G et al (2019) Sleep and pain: recent insights, mechanisms, and future directions in the investigation of this relationship. J Neural Transm. https://doi. org/10.1007/s00702-019-02067-z

20. Gagnon PR (2008) Treatment of delirium in supportive and palliative care. Curr Opin Support Palliat Care 2:60-66. https://doi. org/10.1097/SPC.0b013e3282f4ce05

21. Tate JA, Sereika S, Divirgilio D et al (2013) Symptom communication during critical illness: the impact of age, delirium, and delirium presentation. J Gerontol Nurs 39:28-38. https://doi. org/10.3928/00989134-20130530-03

22. Sirsch E, Lukas A, Drebenstedt C, et al (2019) Pain assessment for older persons in nursing home care: An evidence-based practice guideline. J Am Med Dir Assoc. https://doi:10.1016/j. jamda.2019.08.002. [Epub ahead of print]

23. Herr K, Coyne PJ, Key T et al (2006) Pain assessment in the nonverbal patient: position statement with clinical practice recommendations. Pain Manag Nurs 7:44-52. https://doi.org/10.1016/J. PMN.2006.02.003
24. Hadjistavropoulos T, Voyer P, Sharpe D et al (2008) Assessing pain in dementia patients with comorbid delirium and/or depression. Pain Manag Nurs 9:48-54. https://doi.org/10.1016/J. PMN.2007.12.004

25. American Geriatrics Society Panel on Pharmacological Management of Persistent Pain in Older Persons (2009) Pharmacological management of persistent pain in older persons. J Am Geriatr Soc 57:1331-1346. https://doi.org/10.1111/j.1532-5415.2009.02376.x

26. Lints-Martindale AC, Hadjistavropoulos T, Lix LM, Thorpe L (2012) A comparative investigation of observational pain assessment tools for older adults with dementia. Clin J Pain 28:226-237. https://doi.org/10.1097/AJP.0b013e3182290d90

27. van Dalen-Kok AH, Achterberg WP, Rijkmans WE et al (2018) Pain assessment in impaired cognition (PAIC): content validity of the Dutch version of a new and universal tool to measure pain in dementia. Clin Interv Aging 13:25-34. https://doi.org/10.2147/ CIA.S144651

28. Rijkenberg S, van der Voort PHJ (2016) Can the critical-care pain observation tool (CPOT) be used to assess pain in delirious ICU patients? J Thorac Dis 8:285-287. https://doi.org/10.21037/ jtd.2016.03.32

29. Chookalayia H, Heidarzadeh M, Hassanpour-Darghah $\mathrm{M}$ et al (2018) The critical care pain observation tool is reliable in nonagitated but not in agitated intubated patients. Intensive Crit Care Nurs 44:123-128. https://doi.org/10.1016/J.ICCN.2017.07.012

30. Decker SA (2009) Behavioral indicators of postoperative pain in older adults with delirium. Clin Nurs Res 18:336-347. https://doi. org/10.1177/1054773809341734

31. Gagliese L, Rodin R, Chan V et al (2016) How do healthcare workers judge pain in older palliative care patients with delirium near the end of life? Palliat Support Care 14:151-158. https://doi. org/10.1017/S1478951515000929

32. Fischer T, Hosie A, Luckett T et al (2019) Strategies for pain assessment in adult patients with delirium: a scoping review. J Pain Symptom Manage 58:487-502. https://doi.org/10.1016/J. JPAINSYMMAN.2019.05.020

33. Luckmann R, Vidal A (2010) Design of a handheld electronic pain, treatment and activity diary. J Biomed Inform 43:S32-S36. https://doi.org/10.1016/J.JBI.2010.05.005

34. Atee M, Hoti K, Parsons R, Hughes JD (2017) Pain assessment in dementia: evaluation of a point-of-care technological solution. J Alzheimers Dis 60:137-150. https://doi.org/10.3233/JAD-170375

35. Lautenbacher S, Kunz M (2017) Facial pain expression in dementia: a review of the experimental and clinical evidence. Curr Alzheimer Res 14:501-505

36. Pasero C, McCaffery M (2005) No self-report means no painintensity rating: assessing pain in patients who cannot provide a report. Am J Nurs 105:50-53

37. Pu L, Moyle W, Jones C, Todorovic M (2018) Psychosocial interventions for pain management in older adults with dementia: a systematic review of randomised controlled trials. J Adv Nurs. https://doi.org/10.1111/jan.13929

38. Scottish Intercollegiate Guidelines Network., Scotland. Healthcare Improvement Scotland. Risk reduction and management of delirium: a national clinical guideline

39. Husebo BS, Ballard C, Cohen-Mansfield J et al (2014) The response of agitated behavior to pain management in persons with dementia. Am J Geriatr Psychiatry 22:708-717. https://doi. org/10.1016/J.JAGP.2012.12.006

40. Snow AL, Weber JB, O'Malley KJ et al (2004) NOPPAIN: a nursing assistant-administered pain assessment instrument for use in dementia. Dement Geriatr Cogn Disord 17:240-246. https://doi. org/10.1159/000076446

Publisher's Note Springer Nature remains neutral with regard to jurisdictional claims in published maps and institutional affiliations. 